\section{A tendência da prática da amamentação no Brasil nas décadas de 70 e 80 *}

\section{Breast-feeding trends between 1970 and 1980 in Brazil}

Sonia Isoyama Venancio

Núcleo de Investigação em Saúde da Mulher e da Criança Instituto de Saúde - Secretaria de Estado da Saúde de São Paulo

R. Santo Antonio, 590 - $2^{\circ}$ andar - Bela Vista

01314-000 São Paulo, SP - Brasil

e-mail: NISmc@SAUDE.SP.GOV.BR

\section{Carlos Augusto Monteiro}

Departamento de Nutrição

Faculdade de Saúde Pública da Universidade de São Paulo

Av. Dr. Arnaldo, 715

01246-904 São Paulo, SP - Brasil

E-mail: carlosam@usp.br

* Extraído da dissertação de mestrado "A evolução da prática do aleitamento materno no Brasil nas décadas de 70 e 80 ", apresentada

à Faculdade de Saúde Pública/USP em 12/12/96

\section{Resumo}

A prática da amamentação sofreu um declínio em todo o mundo, levando a conseqüências desastrosas para a saúde das crianças e suas mães. A partir da década de 70 iniciou-se um verdadeiro movimento mundial para o retorno à amamentação, sendo que no Brasil, estudos realizados em algumas cidades indicam o possível sucesso deste movimento no País. Este estudo teve por objetivo descrever a trajetória recente do aleitamento materno no Brasil, em diferentes estratos populacionais, comparando duas pesquisas nacionais (ENDEF/ 75 e PNSN/89). Empregou-se a análise de probitos, que permite estimar freqüências da amamentação a partir de regressões lineares ponderadas, utilizando o teste de aderência de Kolmogorov-Smirnov para verificar a adequação dos modelos obtidos. Verificou-se uma expansão considerável da prática da amamentação no País. Esta tedência ocorreu em todos os estratos da população, porém o aumento da prática da amamentação foi mais acentuado na área urbana, na região Centro-Sul do país, entre as mulheres de maior poder aquisitivo e de maior escolaridade.

Palavras-chave: Aleitamento materno, tendências. Política de saúde. Brasil. 


\section{Abstract}

Breast-feeding practices have declined all over the world, negatively affecting the health of children and mothers. Since the 70's, worldwide activities for reviving breastfeeding were developed, but there are no studies of this trend in Brazil with national representative data. The present study had the objective of describing recent trends of breast-feeding practices in Brazil, in different populational subgroups, by comparing two national surveys carried out in 1975 e 1989. Probit analysis was used to estimate the prevalences of breast-feeding, by linear regression, using the KolmogorovSmirnov test to verify the adequacy of the obtained models. Breast-feeding practices considerably increased in Brazil, specially in the urban area, among women of the central-southern regions of the country, with higher per capita family income, and more years of schooling.

Keywords: Breast-feeding, trends. Health policy. Brazil.

\section{Introdução}

As vantagens da amamentação têm sido amplamente documentadas na literatura. O leite humano é o alimento ideal para o crescimento e desenvolvimento dos lactentes ${ }^{1}$ e confere proteção contra a desnutrição, diarréia, infecções respiratórias, enterocolite necrotizante e septicemia (em prematuros),diminuindo assim a mortalidade infantil. ${ }^{2-9}$

Estudos têm mostrado os benefícios da amamentação exclusiva para crianças que vivem em países desenvolvidos ${ }^{10}$, sendo que nestes, crianças alimentadas com fórmulas infantis têm até cinco vezes mais hospitalizações do que crianças amamentadas. ${ }^{11}$ Além disso, a amamentação ajuda a limitar a fertilidade ${ }^{12}$ e propicia uma importante interação física e psicológica entre mãe e filho. ${ }^{13}$

Por todos estes benefícios, recomendase que as crianças sejam amamentadas exclusivamente até os 4 a 6 meses de vida, e que o aleitamento materno continue pelo menos até dois anos de idade, complementado por outros alimentos. ${ }^{14}$

Apesar da superioridade do leite materno em relação ao leite artificial, o declínio da amamentação é fenômeno conhecido em todo o mundo, especialmente a partir do final do século XIX, com o advento da revolução industrial. ${ }^{15}$

As conseqüências desastrosas do desmame precoce, especialmente nos países em desenvolvimento, levaram a um movimento mundial de retorno à amamentação, que teve início em meados da década de 70, e um momento importante em 1981, quando a Assembléia Mundial de Saúde adotou o Código de Comercialização dos Substitutos do Leite Materno. ${ }^{16}$

Após o despertar da opinião pública européia e norte-americana, várias instituições se mobilizaram no sentido de intensificar as campanhas pró-aleitamento materno em todo o mundo, dentre elas destacando-se a OMS (Organização Mundial da Saúde) e o UNICEF (Fundo das Nações Unidas para a Infância). ${ }^{16}$

Vários estudos realizados nos países 
desenvolvidos apontam para um retorno à prática da amamentação desde a década de $70 .^{17,18}$

Já nos países em desenvolvimento, é a partir da década de 80 que ocorre uma melhora na disponibilidade de dados sobre amamentação, sendo evidenciado um aumento substancial desta prática. ${ }^{19,20}$

No Brasil, apesar da escassez de dados representativos que reflitam a situação da amamentação neste período, estudos regionais mostram que até a década de 70 esta prática sofreu um considerável declínio. ${ }^{21,22}$ Estudos transversais, como o realizado por Sigulem e Tudisco ${ }^{23}$ mostram, por exemplo, que no Município de São Paulo, em meados da década de 70, a duração mediana da amamentação era de aproximadamente um mês. Já a partir da década de 80 , estudos regionais mostram uma tendência de retorno à amamentação..$^{24-28}$

Até o momento, dados de amamentação representativos do país eram disponíveis somente a partir da década de 80. A Pesquisa Nacional sobre Saúde e Nutrição (PNSN/89), estudando crianças menores de 47 meses de idade, revelou que apesar da maioria das crianças iniciar a amamentação, a introdução de outros alimentos era intensa logo nos primeiros dias de vida; a duração da amamentação era de apenas 134 dias e o aleitamento materno, praticado juntamente com a administração de água e chá, tinha uma duração mediana de 72 dias. $^{29}$

A ausência de estimativas nacionais anteriores à década de 80 impossibilitou que se descrevesse a tendência da prática da amamentação no País neste período. Entretanto, verificou-se a possibilidade de obtenção de alguns dados sobre amamentação no ENDEF - Estudo Nacional da Despesa Familiar, pesquisa realizada pelo Instituto Brasileiro de Geografia e Estatística (IBGE) em meados da década de 70, em 55 mil domicílios do país.

Este estudo tem por objetivo, portanto, disponibilizar dados sobre a trajetória recente do aleitamento materno no Brasil, comparando dados das décadas de 70 e 80 segundo área de residência (urbana ou rural), macro-regiões (Norte, Nordeste, Centro-Oeste, Sudeste e Sul) e indicadores socioeconômicos (renda familiar per capita e escolaridade materna).

\section{Metodologia}

O estudo utilizou dados de duas pesquisas nacionais, a Pesquisa Nacional sobre Saúde e Nutrição - PNSN (realizada em 1989 pelo Instituto Nacional de Alimentação e Nutrição) e o Estudo Nacional da Despesa Familiar - ENDEF (realizado em 1974-1975, pelo IBGE).

Em face da inexistência de dados detalhados sobre a alimentação infantil no inquérito nacional realizado na década de 70 , este trabalho circunscreveu-se à análise da tendência da prática da amamentação, independente do recebimento ou não de outros alimentos. Os indicadores utilizados foram a freqüência de crianças amamentadas com 1, 2, 3, 4, 6, e 12 meses de idade e a duração mediana da amamentação.

Em ambos os arquivos de dados, estudou-se a situação de todas as crianças menores de um ano quanto à prática do aleitamento materno (7591 crianças no banco de dados do ENDEF e 1431 crianças na PNSN).

Na PNSN a condição atual das crianças quanto à amamentação foi verificada a partir da aplicação de um questionário alimentar. No ENDEF buscava-se avaliar o consumo alimentar da família como um todo, não havendo no questionário da pesquisa qualquer menção à ingestão ou não de leite materno. Entretanto, todas as mulheres, incluindo as mães de crianças menores de um ano, eram indagadas se estavam grávidas ou amamentando. A partir desta questão resgatou-se o "status" relativo à amamentação dos menores de um ano estudados pelo ENDEF.

As seguintes variáveis foram utilizadas: identificação da criança, idade da criança, 
área (urbana ou rural), escolaridade da mãe (analfabeta, até 4 anos de escolaridade e mais de 4 anos), renda familiar per capita (em quartis) e fator de expansão da amostra.

Neste estudo, seguindo a proposta de Ferreira e col. ${ }^{30}$ foram utilizados somente dados atuais da amamentação ("current status"), a fim de evitar o "recall bias", decorrente de informações em geral imprecisas, fornecidas pelas mães sobre a alimentação das crianças. Empregou-se a análise de probitos para a obtenção das freqüências da amamentação em idades exatas. O modelo de probitos permite estimar essas freqüências a partir de regressões lineares ponderadas, considerando a amostra total de crianças em estudo. Deste modo, os resultados são menos influenciados pelas flutuações amostrais que ocorrem mês a mês.

A análise de probitos, descrita por Finney $^{31}$, estima a proporção de crianças que apresentarão o desmame através de uma regressão linear ponderada de probitos (y) em relação às idades $(\mathrm{x})$, utilizando o maximum likelihood iterative procedure. A relação entre probitos (y) e valores de idade (x) é dada pela equação: $\mathrm{y}=5+(\mathrm{x}-\mu) / \mathrm{s}$ onde $\mathrm{x}=$ idade média, $\mu=$ média da distribuição hipotética de idades de desmame em uma dada população e s= desvio-padrão.

Os cálculos foram feitos através de um pacote estatístico elaborado por Ferreira ${ }^{30}$ em linguagem BASIC. O teste de aderência de Kolmogorov-Smirnov foi utilizado para verificar o ajuste dos modelos obtidos, adotando-se um $\alpha$ de $5 \% .{ }^{30}$

\section{Resultados}

\section{A tendência da amamentação no País}

Considerando-se o conjunto das crianças estudadas pelos dois inquéritos, verificou-se uma expansão considerável da prática da amamentação no País, em todas as idades, sendo que a duração mediana da amamentação foi mais do que duplicada entre 1975 e 1989, passando de 2,5 meses para 5,5 meses (Tabela 1).

Tabela 1 - Percentagens de crianças amamentadas em diferentes idades (e respectivos intervalos de confiança). Brasil, áreas urbana e rural, 1975 e 1989.

Table 1 - Percentage of breastfed children at different ages (and respective confidence intervals).

Brazil, urban and rural areas, 1975 and 1989.

\begin{tabular}{lcccccc}
\hline & \multicolumn{2}{c}{ Brasil } & \multicolumn{2}{c}{ Urbano } & \multicolumn{2}{c}{ Rural } \\
$\begin{array}{l}\text { \% de crianças } \\
\text { amamentadas }\end{array}$ & 1975 & 1989 & 1975 & 1989 & 1975 & 1989 \\
\hline 1 mês & 66 & 79 & 57 & 79 & 74 & 87 \\
& $(61-70)$ & $(66-88)$ & $(53-62)$ & $(66-89)$ & $(67-80)$ & $(81-92)$ \\
2 meses & 53 & 68 & 43 & 68 & 64 & 77 \\
& $(50-56)$ & $(59-77)$ & $(40-46)$ & $(58-77)$ & $(59-69)$ & $(72-82)$ \\
3 meses & 46 & 62 & 35 & 61 & 57 & 70 \\
& $(43-48)$ & $(54-69)$ & $(32-37)$ & $(52-69)$ & $(53-61)$ & $(65-74)$ \\
4 meses & 40 & 57 & 29 & 55 & 52 & 64 \\
6 meses & $(38-43)$ & $(50-63)$ & $(27-31)$ & $(48-63)$ & $(49-56)$ & $(60-68)$ \\
& 33 & 49 & 22 & 47 & 46 & 56 \\
12 meses & $(31-36)$ & $(42-56)$ & $(20-24)$ & $(40-55)$ & $(42-49)$ & $(52-60)$ \\
& 23 & 37 & 13 & 34 & 34 & 41 \\
\hline Duração mediana & 74 & 167 & $(11-15)$ & $(25-45)$ & $(29-40)$ & $(35-47)$ \\
(dias) & $(63-85)$ & $(110-266)$ & $(37-53)$ & $(101-227)$ & $(108-163)$ & $(190-283)$ \\
\hline
\end{tabular}


A tendência da amamentação nas áreas urbana e rural

Observaram-se aumentos nas percentagens de crianças amamentadas em todas as idades, tanto na área urbana quanto na área rural (Tabela 1), verificando-se, porém, que o incremento destas percentagens foi mais acentuado na área urbana.

A diferença entre as medianas de amamentação das duas áreas diminuiu, já que na área urbana a mediana da amamentação mais que triplicou (de 45 passou a 150 dias), enquanto na área rural não chegou a duplicar (de 133 passou a 227 dias).

\section{A tendência da amamentação nas macro- regiões}

A comparação dos dados de 1975 e 1989 mostrou um aumento da duração mediana da amamentação nas regiões Nordeste, Centro-Oeste, Sudeste e Sul (Tabela 2)*.

Os incrementos da duração mediana da amamentação foram maiores nas regiões Centro-Oeste e Sudeste, onde aumentos de
3,2 e 2,8 vezes foram evidenciados. Os incrementos menos intensos ocorreram nas regiões Sul, onde a mediana duplicou e Nordeste, onde o aumento foi de 1,7 vezes.

\section{A tendência da amamentação segundo a renda}

Observou-se um aumento das percentagens de crianças amamentadas em todas as categorias de renda no País (Tabela 3), sendo este mais acentuado entre as crianças de maior renda. As crianças mais pobres continuaram sendo as que apresentam maior duração da amamentação, porém a diferença entre os grupos extremos de renda diminuiu consideravelmente (a duração da amamentação no grupo de crianças mais pobres, que era 4 vezes maior que a das crianças mais ricas, não chega a ser o dobro em 1989). Desta forma, a relação entre a duração mediana da amamentação e a renda, que era inversamente linear em 1975, deixou de apresentar esta característica.

Tabela 2 - Percentagens de crianças amamentadas em diferentes idades (e respectivos intervalos de confiança) segundo macro-regiões. Brasil,1975 e 1989.

Table 2 - Percentage of breastfed children at different ages (and respective confidence intervals) according to regions. Brazil, 1975 and 1989.

\begin{tabular}{lcccccccc}
\hline & \multicolumn{3}{c}{ Nordeste } & \multicolumn{2}{c}{ Centro-Oeste } & \multicolumn{2}{c}{ Sudeste } & \multicolumn{2}{c}{ Sul } \\
\% de crianças & 1975 & 1989 & 1975 & 1989 & 1975 & 1989 & 1975 & 1989 \\
amamentadas & & & & & & & & \\
\hline 1 mês & 70 & 83 & 65 & 89 & 62 & 82 & 66 & 83 \\
& $(65-75)$ & $(67-94)$ & $(55-75)$ & $(61-98)$ & $(56-69)$ & $(64-93)$ & $(57-74)$ & $(59-95)$ \\
2 meses & 58 & 71 & 50 & 85 & 49 & 71 & 55 & 72 \\
& $(54-61)$ & $(57-82)$ & $(42-57)$ & $(58-97)$ & $(44-54)$ & $(57-83)$ & $(49-61)$ & $(54-86)$ \\
3 meses & 50 & 62 & 40 & 79 & 41 & 63 & 48 & 65 \\
& $(47-53)$ & $(51-72)$ & $(35-46)$ & $(54-94)$ & $(38-45)$ & $(52-74)$ & $(43-53)$ & $(50-77)$ \\
4 meses & 44 & 55 & 34 & 74 & 36 & 58 & 43 & 59 \\
& $(41-47)$ & $(46-65)$ & $(29-39)$ & $(51-89)$ & $(33-39)$ & $(47-67)$ & $(39-48)$ & $(46-71)$ \\
6 meses & 37 & 46 & 26 & 59 & 29 & 49 & 37 & 51 \\
& $(34-40)$ & $(36-56)$ & $(22-30)$ & $(39-76)$ & $(26-32)$ & $(39-59)$ & $(32-42)$ & $(38-63)$ \\
12 meses & 25 & 30 & 15 & 17 & 19 & 35 & 26 & 36 \\
& $(22-29)$ & $(19-43)$ & $(10-20)$ & $(3-49)$ & $(15-22)$ & $(22-49)$ & $(21-32)$ & $(21-55)$ \\
\hline Duração mediana & 92 & 157 & 65 & 208 & 60 & 167 & 87 & 177 \\
(dias) & $(78-106)$ & $(93-276)$ & $(46-85)$ & $(75-420)$ & $(47-75)(100-304)$ & $(59-116)$ & $(83-562)$ \\
\hline
\end{tabular}

* Análises preliminares dos dados obtidos na Região Norte em 1975 indicaram falta de consistência nas informações relativas à condição de amamentação das crianças, razão pela qual optou-se pelo não estudo individualizado da evolução da amamentação nesta região. Menos do que $5 \%$ da população brasileira de menores de um ano vivem nas áreas urbanas da região Norte, as únicas estudadas pelo ENDEF e pela PNSN, motivo pelo qual a possível pior qualidade das pesquisas nesta região não deve influenciar de modo importante as estimativas nacionais sobre a amamentação no país. 
Tabela 3 - Percentagens de crianças amamentadas em diferentes idades (e respectivos intervalos de confiança) segundo quartis da renda familiar per capita. Brasil, 1975 e 1989. Table 3 -Percentage of breastfed children at different ages (and respective confidence intervals), according to per capita income. Brazil, 1975 and 1989.

\begin{tabular}{|c|c|c|c|c|c|c|c|c|}
\hline \multirow[b]{2}{*}{$\begin{array}{l}\% \text { de crianças } \\
\text { amamentadas }\end{array}$} & \multicolumn{2}{|c|}{$\begin{array}{c}\text { Renda } 1 \\
1^{\circ} \text { quartil }\end{array}$} & \multicolumn{2}{|c|}{$\begin{array}{l}\text { Renda } 2 \\
2^{\circ} \text { quartil }\end{array}$} & \multicolumn{2}{|c|}{$\begin{array}{c}\text { Renda } 3 \\
3^{\circ} \text { quartil }\end{array}$} & \multicolumn{2}{|c|}{$\begin{array}{c}\text { Renda } 4 \\
4^{\circ} \text { quartil }\end{array}$} \\
\hline & 1975 & 1989 & 1975 & 1989 & 1975 & 1989 & 1975 & 1989 \\
\hline \multirow[t]{2}{*}{1 mês } & 75 & 85 & 73 & 81 & 61 & 75 & 49 & 88 \\
\hline & $(69-80)$ & $(65-95)$ & $(66-79)$ & $(72-88)$ & $(53-67)$ & $(53-90)$ & $(43-54)($ & $(65-256)$ \\
\hline \multirow[t]{2}{*}{2 meses } & 64 & 76 & 60 & 71 & 47 & 64 & 34 & 75 \\
\hline & $(60-68)$ & $(61-88)$ & $(55-65)$ & $(63-77)$ & $(42-52)$ & $(48-78)$ & $(31-38)$ & $(55-89)$ \\
\hline \multirow[t]{2}{*}{3 meses } & 58 & 70 & 53 & 64 & 39 & 57 & 26 & 64 \\
\hline & $(54-61)$ & $(58-81)$ & $(49-57)$ & $(58-70)$ & $(35-43)$ & $(44-69)$ & $(24-29)$ & $(48-78)$ \\
\hline \multirow[t]{2}{*}{4 meses } & 53 & 66 & 47 & 59 & 34 & 52 & 22 & 56 \\
\hline & $(50-56)$ & $(55-75)$ & $(43-51)$ & $(53-64)$ & $(31-38)$ & $(40-63)$ & $(19-24)$ & $(41-69)$ \\
\hline \multirow[t]{2}{*}{6 meses } & 46 & 59 & 39 & 51 & 27 & 44 & 16 & 43 \\
\hline & $(43-49)$ & $(48-69)$ & $(36-43)$ & $(46-57)$ & $(24-31)$ & $(33-56)$ & $(14-18)$ & $(30-57)$ \\
\hline \multirow[t]{2}{*}{12 meses } & 35 & 46 & 27 & 38 & 17 & 32 & 8 & 24 \\
\hline & $(30-39)$ & $(31-61)$ & $(23-32)$ & $(31-46)$ & $(14-21)$ & $(19-49)$ & $(7-10)$ & $(11-43)$ \\
\hline Duração & 137 & 254 & 103 & 177 & 58 & 137 & 31 & 153 \\
\hline $\begin{array}{l}\text { mediana } \\
\text { (dias) }\end{array}$ & $(115-162)$ & $(134-267)$ & (84-122) & $(133-248)$ & $(42-73)$ & $(50-354)$ & $(23-38)$ & $(91-255)$ \\
\hline
\end{tabular}

A tendência da amamentação segundo a escolaridade da mãe.

Houve um aumento das percentagens de crianças amamentadas e da duração da amamentação nos três grupos de escola- ridade da mãe (Tabela 4), porém, este aumento foi mais expressivo entre mulheres de maior escolaridade. Neste grupo, a duração da amamentação que era de 46 dias mais que triplicou, passando para 159 dias.

Tabela 4 - Percentagens de crianças amamentadas em diferentes idades (e respectivos intervalos de confiança) segundo a escolaridade da mãe. Brasil,1975 e 1989.

Table 4-Percentage of breastfed children at different ages (and respective confidence intervals). according to mother's schooling. Brazil,1975 and 1989.

\begin{tabular}{lcccccc}
\hline \multirow{2}{*}{$\begin{array}{l}\text { \% de crianças } \\
\text { amamentadas }\end{array}$} & \multicolumn{2}{c}{ Analfabetas } & \multicolumn{2}{c}{$1-4$ anos } & \multicolumn{2}{c}{$>$ anos } \\
\cline { 2 - 7 } & 1975 & 1989 & 1975 & 1989 & 1975 & 1989 \\
\hline 1 mês & 69 & 93 & 66 & 77 & 59 & 83 \\
& $(64-74)$ & $(84-98)$ & $(61-70)$ & $(67-86)$ & $(38-79)$ & $(71-91)$ \\
2 meses & 60 & 81 & 52 & 68 & 35 & 71 \\
& $(56-63)$ & $(71-88)$ & $(48-55)$ & $(60-75)$ & $(22-49)$ & $(62-80)$ \\
3 meses & 54 & 70 & 43 & 62 & 22 & 63 \\
& $(50-56)$ & $(61-77)$ & $(40-46)$ & $(56-68)$ & $(14-33)$ & $(55-70)$ \\
4 meses & 49 & 60 & 37 & 58 & 15 & 57 \\
& $(46-52)$ & $(53-68)$ & $(35-40)$ & $(52-64)$ & $(9-24)$ & $(50-63)$ \\
6 meses & 43 & 46 & 30 & 51 & 8 & 47 \\
& $(40-46)$ & $(38-55)$ & $(27-32)$ & $(45-58)$ & $(4-16)$ & $(41-54)$ \\
12 meses & 33 & 24 & 18 & 40 & 2 & 32 \\
& $(30-38)$ & $(14-36)$ & $(16-21)$ & $(32-49)$ & $(1-7)$ & $(24-42)$ \\
\hline Duração mediana & 115 & 159 & 67 & 188 & 46 & 159 \\
(dias) & $(94-138)$ & $(128-206)$ & $(57-77)$ & $(130-312)$ & $(24-67)$ & $(116-222)$ \\
\hline
\end{tabular}


Já entre as analfabetas o aumento foi mais discreto, passando de 115 para 159 dias. Desta forma, enquanto que em 1975 a relação entre a duração da amamentação e a escolaridade da mãe era linear inversa, em 1989 o comportamento das mulheres brasileiras analfabetas e com 4 anos ou mais de escolaridade é semelhante.

\section{Discussão}

Este estudo, ainda que retratando a tendência temporal de apenas duas décadas, confirma a hipótese de aumento da frequência e duração da amamentação no País, por meio da análise de dois inquéritos representativos da população infantil brasileira.

É interessante verificar que o retorno à prática da amamentação neste período ocorreu em todos os estratos da população, ou seja, entre as crianças residentes em áreas urbanas e rurais, de qualquer região do País, filhos de mães analfabetas e das que atingiram graus maiores de escolaridade, de famílias pobres e ricas.

É importante destacar que a tendência ascendente da amamentação no País neste período, sumarizada por um aumento de cerca de três meses em sua duração mediana, foi mais acentuada no Brasil do que em vários países latino-americanos. Com base na análise de inquéritos populacionais realizados nas décadas de 70 e 80 , Pérez-Escamilla ${ }^{32}$ identifica ascensões mais modestas da prática da amamentação no Equador ( $+0,3$ meses), Peru (+1,3 meses) e Trinidad e Tobago ( $+2,9$ meses), estagnação no México e retrocesso na Colômbia ( $-0,3$ meses) e República Dominicana (-1,3 meses). Mesmo assim, o Brasil apresentava, no final da década de 80 , duração média da amamentação inferior à observada nos países latino-americanos.

Analisando a duração da amamentação nas áreas urbanas e rurais, verificou-se que a diferença entre área urbana e rural diminuiu substancialmente, apesar de as mulheres da área rural continuarem amamentando por mais tempo. Embora o aumento da amamentação tenha sido menos expressivo na área rural, as taxas de amamentação nessa região nunca chegaram a atingir níveis tão baixos quanto na área urbana. Este fato pode indicar que houve, de certa forma, uma reversão da tendência esperada de assimilação de valores culturais dominantes por parte dos estratos da população menos favorecidos. ${ }^{17}$

É interessante observar que, apesar de as mulheres com renda familiar per capita menor ainda amamentarem por mais tempo, o aumento da duração da amamentação foi mais acentuado entre mulheres de melhor situação socioeconômica, de tal forma que a duração da amamentação em 1989 foi semelhante entre as mulheres analfabetas e as que estudaram mais de 4 anos. Considerando que o analfabetismo materno constituiu-se em um importante fator de risco para a morbi-mortalidade infantil, ${ }^{33}$ os programas de incentivo à amamentação devem priorizar intervenções no sentido de promover a amamentação neste grupo.

Vale notar que pesquisa nacional realizada em 1996 pela Sociedade Civil BemEstar Familiar - BEMFAM indica que prossegue, nos anos 90, a tendência de aumento na prática da amamentação no País. Monteiro, ${ }^{34}$ ao comparar resultados da referida pesquisa com inquérito semelhante realizado pela mesma BEMFAM, em 1986, identifica aumento da amamentação em todas as idades e regiões do país, sendo particularmente notável o aumento registrado na região Nordeste (Tabela 5). Porém, esses dados mais recentes revelam que estamos longe de atingir as metas da Cúpula Mundial pela Infância, relativas à amamentação. ${ }^{35}$

A compreensão do comportamento favorável das mulheres brasileiras em relação à amamentação, ocorrido com maior ou menor intensidade em todos os estratos da população, passa pela análise do contexto sociopolítico-cultural do País nas últimas décadas, em especial das políticas governamentais pró-amamen- 
Tabela 5 - Percentagens de crianças amamentadas em diferentes faixas etárias.

Brasil e região Nordeste, 1986 e 1996.

Table 5 - Percentage of breastfed children in

different age intervals. Brazil and Northeast, 1986 and 1996.

\begin{tabular}{lcccc}
\hline & \multicolumn{2}{c}{ Brasil } & \multicolumn{2}{c}{ Nordeste } \\
\cline { 2 - 5 } Faixa etária & 1986 & 1996 & 1986 & 1996 \\
\hline $0-4$ meses & 73,5 & 85,4 & 66,7 & 85,4 \\
10-14 meses & 27,5 & 37,1 & 24,2 & 33,3 \\
22-26 meses & 10,2 & 15,5 & 6,0 & 13,4 \\
\hline
\end{tabular}

tação, iniciadas na década de 80 .

Destaca-se a criação, em 1981, do PNIAM (Programa Nacional de Incentivo ao Aleitamento Materno) que iniciou suas atividades com o lançamento de uma extensa campanha na imprensa falada e escrita. ${ }^{36}$

Embora haja evidência de que a tendência de aumento da prática da amamentação no Brasil possa ter se iniciado em um momento anterior à criação do PNIAM $^{37}$, talvez decorrente do movimento internacional pró-amamentação surgido na década de 70, é inegável que apenas a partir de 1981 políticas institucionais consistentes de promoção e apoio à amamentação são implantadas no País.

Cabe citar a aprovação de leis, como a do alojamento conjunto, em 1983, que tornou sua prática obrigatória através de portaria do Ministério da Saúde; da Norma Brasileira para Comercialização de Alimentos para Lactentes, aprovada pelo Conselho Nacional de Saúde em 1988; da extensão da licença-maternidade para 120 dias e a criação da licença-paternidade de 5 dias, na Constituição de $1988 .{ }^{38,39}$

O Brasil aderiu, ainda, a políticas internacionais pró-amamentação, assinando a Declaração de Innocenti ${ }^{14}$, incorporando a Iniciativa Hospital Amigo da Criança - IHAC da OMS/UNICEF ${ }^{40}$ às atividades do PNIAM, e promovendo anualmente, no mês de agosto, a Semana Mundial da Amamentação.

O PNIAM tem desenvolvido também atividades de treinamento de recursos humanos, através dos seis centros de referência em aleitamento materno existentes no país. ${ }^{41}$

É preciso analisar, porém, por que o retorno à amamentação, embora comprovadamente desencadeado em todas as classes sociais, não se dá em um ritmo mais acelerado, tendo em vista as políticas pró-amamentação adotadas no País.

Deve-se considerar, inicialmente que a alimentação artificial tornou-se não somente uma opção para as mulheres, mas uma prática amplamente utilizada e culturalmente aceita. Neste contexto, a prática da amamentação precisa ser socialmente reconstruída. O que se vê, entretanto, é que as estruturas sociais necessárias para esta reconstrução parecem não ser, no momento, suficientemente sólidas. Atualmente, vive-se uma situação em que muitas são as ambigüidades no tocante à criação de condições favoráveis à prática da amamentação.

Infrações à Norma Brasileira são ainda detectadas; muitas mulheres desconhecem a legislação trabalhista que assegura seus direitos; profissionais de saúde ainda orientam o desmame precoce e a introdução de leites artificiais na dieta da criança; maternidades continuam adotando rotinas desfavoráveis ao estabelecimento da amamentação; os treinamentos realizados parecem ter impacto limitado sobre mudanças nas rotinas das institutições. ${ }^{42-44}$

$\mathrm{O}$ retorno à amamentação, segundo Palmer ${ }^{45}$, passa pela devolução da amamentação às mulheres. Nesse sentido, uma estratégia importante para fazer ressurgir a amamentação é a formação de grupos de mães para atuar junto aos serviços de saúde e na comunidade, prática até então pouco difundida em nosso meio. ${ }^{46}$

Em 1996, a V Semana Mundial da Amamentação discutiu o tema "Amamentação, uma responsabilidade de todos”. Parece estar aí a chave do sucesso da amamentação: somente a atitude coerente do governo, dos profissionais e serviços de saúde, dos empregadores, das famílias, das organizações não-governamentais, enfim, da socie- 
dade como um todo e a construção de novos atores sociais que atuem no palco de uma nova "cultura da amamentação", poderão recriar o ato de amamentar como uma prática que beneficiará a maioria das mulheres e crianças brasileiras.

\section{Summary}

Breast-feeding declined all over the world, negatively affecting the health of children and mothers. Since the 70s worldwide activities for reviving breast-feeding were developed, but there are no studies of this trend in Brazil, with national representative data. The present study had the objective of describing recent trends in breast-feeding in Brazil, in different populational subgroups, by comparing two national surveys carried out in 1975 and 1989. Probit analysis was used to estimate the prevalences of breastfeeding from linear regression, using the Kolmogorov-Smirnov test to verify the appropriateness of the models obtained. Breast-feeding practices have increased considerably in Brazil: its median duration doubled between 1975 and 1989 (from 2,5 to 5,5 months). The increase in the median duration of breast-feeding was larger (from
45 to 150 days) in the urban area, consequently decreasing differences between urban and rural areas (where the median duration changed from 133 to 227 days). The median duration of breast-feeding increased three-fold in central and southern regions (medians of 208 and 167 days respectively) and had the poorest increase (from 92 to 157 days) in the northeastern region. There was a larger increase of breastfeeding in the group of children with higher family income (median from 31 to 153 days) but the differences between rich and poor children (median from 137 to 254 days) also decreased. The median duration of breastfeeding was 46 days in the group of mothers with more years of schooling, and increased three-fold. On the other hand, duration of breast-feeding increased only from 155 to 159 days among illiterate mothers.

\section{Referências}

1. Akré J. Infant feeding: the physiological basis. Bull World Health Organ 1989; 67 Suppl: 1-108.

2. Feachem RG, Koblinsky MA. Interventions for the control of diarrhoeal diseases among young children: promotion of breastfeeding._Bull World Health Organ 1984; 62: 271-91.

3. Victora C G, Vaughan JP, Lombardi C, Fuchs SMC, Gigante L.P, Smith PG, Nobre LC, Teixeira AMB, Moreira LB, Barros FC. Evidence for protection by breastfeeding against infant deaths from infectious diseases in Brazil. Lancet 1987; 2: 319-22.

4. Victora C G, Smith PG, Barros FC, Vaughan JP, Fuchs SC. Risk factors for deaths due to respiratory infections among Brazilian infants. Int J Epidemiol 1989; 18: 918-25.

5. Victora C, Huttley SR, Fuchs SC, Nobre LC, Barros FC. Deaths due to dysentery, acute and persistent diarrhoea among Brazilian infants. Acta Paediatr 1992; 381 Suppl: 7-11.

6. Monteiro CA, Rea M, Victora C. Can infant mortality be reduced by promoting breastfeeding? Evidence from São Paulo city. Health Policy Plan 1990; 5: 23-9.

7. Lucas A, Cole TJ. Breast milk and neonatal necrotizing enterocolitis. Lancet 1990; 336:1519-23.
8. Ashraf RN, Jahil F, Zaman S, Karlberg J, Khan SR, Lindblad BS, Hanson LA. Breastfeeding and protection against neonatal sepsis in a high risk population. Arch Dis Child 1991; 66: 488-90.

9. Woolridge MW, Phil D, Baum JD. Recent advances in breast feeding. Acta Paediatr Jpn 1993; 35: 1-12.

10. Ford K, Labbok M. Breastfeeding and child health in United States. J Biosoc Sci 1993; 25: 187-94.

11. De Zoysa I, Rea MF, Martines J. Why promote breastfeeding in diarrhoeal disease control programmes? Health Policy Plan 1991; 6: 371-9.

12. Kennedy KI, Visness CM. Contraceptive efficacy of lactational amenorrhoea. Lancet 1992; 339: 227-30.

13. Harfouche JK. Psycho-social aspect of breastfeeding including bonding. Food Nutr Bull 1980; 2: 2-6.

14. World Health Organization. Innocenti Declaration on the protection, promotion and support of breastfeeding. Ecol Food Nutr 1991; 26: 271-3.

15. Palmer G. The politics of breastfeeding. $4^{\text {th }} \mathrm{ed}$. London: Pandor Press; 1993.

16. Orlandi O. Teoria e prática do amor à criança: 
introdução à pediatria social no Brasil. Rio de Janeiro: Jorge Zahar; 1985.

17. World Health Organization. The prevalence and duration of breastfeeding: a critical review of available information. World Health Stat $Q$ 1982; 35: 92-116.

18. Helsing E, Saadeh R. Breastfeeding trends in a global perspective. News Health Care Dev Countries 1991; 3: 4-9, 1991.

19. Sharma RK, Rutstein SO. Comparative analysis of the determinants of infant feeding practices. Washington (DC): IRD/Macro International; 1991. [Proceedings of the Demographic and Health Surveys World Conference].

20. Trussel J, Grummer-Strawn L, Rodriguez G, Vanlandingham M. Trends and differentials in breastfeeding behaviour: evidence from WFS and DHS. Popul Stud 1992; 46: 285-307.

21. Sousa PLR, Barros FC, Pinheiro GNM, Gazzalle RV. The decline in breast-feeding in Brazil. $J$ Trop Pediatr Environ Child Health 1975; 21: 212-3.

22. Zuniga HPP, Monteiro CA. Uma nova hipótese para a ascensão da mortalidade infantil da cidade de São Paulo nos anos 60. In: Monteiro, C.A. Velhos e novos males da saúde no Brasil: a evolução do país e de suas doenças. São Paulo: HUCITEC/ABRASCO/ NUPENS-USP; 1995. p. 157-73.

23. Sigulem DM, Tudisco ES. Aleitamento natural em diferentes classes de renda no Município de São Paulo. Arch Latinoam Nutr 1980; 30: 400-16.

24. Monteiro CA, Zuniga HPP, Benício MH, Rea MF, Tudisco ES, Sigulem DM. The recent revival of breast-feeding in the City of São Paulo, Brazil. Am J Public Health 1987; 77: 964-6.

25. Rea M. The Brazilian national breastfeeding programme: a succssesful history. Int J Gynaecol Obstet 1990; 31 suppl. 1: 79-82.

26. Rea MF, Berquó E. Impact of the Brazilian national breast-feeding programme on mothers in greater São Paulo. Bull World Health Organ 1990; 68: 36571.

27. Martins Filho J., Sanged CAA. Modificação da prevalência da amamentação na região de Campinas, após oito anos de estímulo contínuo, em nível ambulatorial. J Pediatr 1987; 62: 251-6.

28. Santos TF. Amamentação e mortalidade infantil no Nordeste. In: Sociedade Civil Bem-Estar Familiar. Pesquisa sobre saúde familiar no Nordeste. Rio de Janeiro: 1994.

29. Leão MM, Coitinho DC, Recine E, Costa LAL, Lacerda AJ O perfil do aleitamento materno no Brasil. In: Fundação IBGE/UNICEF. Perfil estatístico de crianças e mães no Brasil . Aspectos de saúde e nutrição de crianças no Brasil, 1989. Rio de Janeiro: IBGE; 1992.p. 97-110.
30. Ferreira MU, Cardoso MA, Santos ALS, Ferreira CS, Szarfarc SC. Rapid epidemiologic assesment of breastfeeding practices: probit analysis of current status data. J Trop Pediatr 1995; 41: 5-8.

31. Finney DJ. Probit analysis. $3^{\text {rd }}$ ed. Cambridge: Cambridge University Press; 1980.

32. Pèrez-Escamilla R. Patrones de la lactancia natural en América Latina y el Caribe. Bol Oficina Sanit Panam 1993; 115: 185-93.

33. BEMFAM - Sociedade Civil Bem-Estar Familiar no Brasil. Pesquisa Nacional sobre Demografia e Saúde, 1996. Rio de Janeiro; 1997.

34. Monteiro CA. O panorama da nutrição infantil nos anos 90. Brasília: UNICEF; 1997. (Cadernos de Políticas Sociais, Série Documentos para Discussão $1)$.

35. Organización Panamericana de la Salud. La salud maternoinfantil: metas para 1995 e indicadores para el seguimento. Washington DC; 1990.

36. Matthai J. The Brazilian national breast-feeding programme. Assigment Child 1983; 61/62: 225-4.

37. Venancio SI. A evolução da prática do aleitamento materno no Brasil nas décadas de 70 e 80. São Paulo: 1996. [Dissertação de mestrado - Faculdade de Saúde Pública da USP].

38. Brasil. Constituição 1988. Constituição; República Federativa do Brasil. Brasília: Senado Federal; 1988.

39. Ministério da Saúde/INAN. Conselho Nacional de Saúde. Norma brasileira para comercialização de alimentos para lactentes. Brasília: 1993.

40. Organização Mundial da Ssaúde. Proteção, promoção e apoio ao aleitamento materno: o papel especial dos serviços de saúde. Genebra, 1989.

41. Instituto Nacional de Alimentação e Nutrição. Relatório de atividades. Brasília: PNIAM; 1996.

42. Toma T S. Violando a norma 1996: relatório nacional das violações à normabrasileira para comercialização de alimentos para lactentes. São Paulo: IBFAN/UNICEF/IS; 1996.

43. Rea MF, Venancio SI, Batista LE, Santos RG, Greiner T. Possibilidades e limitações da amamentação entre mulheres trabalhadoras formais. Rev Saúde Pública 1997; 31: 149-56.

44. Westphal MF, Taddei JAC, Venancio SI, Bógus CM. Breast-feeding training for health professionals and resultant institutional changes. Bull World Health Organ 1995; 73: 461-8.

45. Palmer G. Give breastfeeding back to the mothers. J Hum Lact 1991; 7: 1-2.

46. Kyenkya-Isabirye M, Magalhães R. The mothers'support groups role in the health care system. Int J Gynaecol Obstet 1990; 31 Suppl1: 85-90. 